\title{
Homopolymer Micelles in Heterogeneous Solvent Mixtures
}

\author{
Subhadeep Basu, Dharma Rao Vutukuri, and S. Thayumanavan \\ Department of Chemistry, University of Massachusetts, Amherst, Massachusetts 01003, \\ thai@chem.umass.edu
}

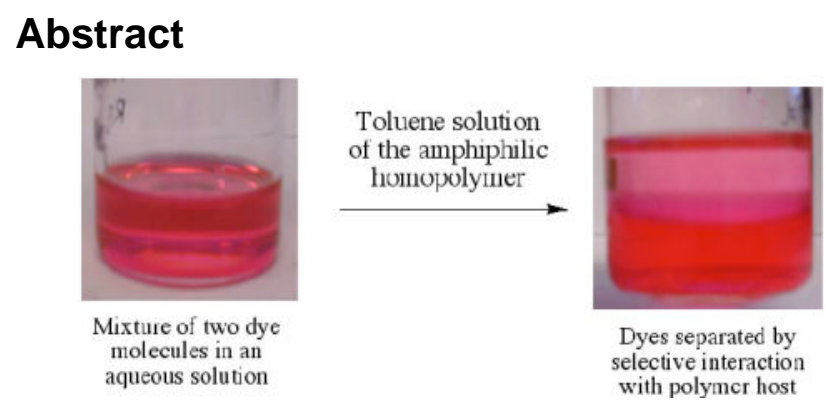

Amphiphilic homopolymers containing a hydrophilic and a hydrophobic functionality in each monomer unit have been shown to form polar or apolar containers depending on the solvent environment. When presented with a mixture of solvents, these polymeric containers are capable of releasing certain guest molecules. The fundamental mechanism behind these properties is investigated and the utility of these assemblies in separations has been demonstrated with an example.

Designing artificial scaffolds to bind guest molecules using specific interactions is at the heart of supramolecular chemistry. ${ }^{1}$ Amphiphilic assemblies, such as micelles, use solvophobic interactions as the primary driving force for binding. ${ }^{2}$ For example, micelles are capable of sequestering lipophilic guest molecules in water, which are otherwise insoluble. ${ }^{3}$ Similarly, inverse micelles are capable of sequestering hydrophilic guest molecules in organic solvents.

${ }^{4}$ We have recently reported a new class of amphiphilic polymers that presents their functionalities in a solvent-dependent fashion that are reminiscent of micelles in water and inverted micelles in an apolar solvent. ${ }^{5}$ While one would expect these polymers to behave like a micellar host or an inverse micellar host in a polar or an apolar solvent respectively, what would be the behavior of these assemblies in a heterogeneous mixture of both solvents? This is especially interesting with these polymers, because these are known to form organized assemblies in both polar and aopolar solvents. Would these assemblies disintegrate at the interface and find a thermodynamically preferable solvent or would these be stable in the solvents they are initially assembled in?

In order to address these questions, we synthesized polymers $\mathbf{1 - 5}$ shown in Chart 1 . These polymers differ from each other in the lipophilic side chain, since we thought it is useful to study the effect of hydrophilic-lipophilic balance on the container properties of the amphiphilic assemblies formed from these homopolymers. All polymers were synthesized from the corresponding styrene-based monomer using the nitroxide mediated polymerization method. 6,7

Based on our prior report on the spatial disposition of the amphiphilic functionalities in these assemblies, ${ }^{5}$ one would expect these assemblies to bind to lipophilic guest molecules in water 
and hydrophilic guest molecules in an apolar solvent such as toluene. However, it is useful to confirm that the polymers do exhibit these properties, before discussing the behavior of these assemblies in solvent mixtures.

Reichardt's dye (RD, pyridinium-N-phenoxide betaine $)^{8}$ is a lipophilic dye molecule, the solvatochromic behavior of which spans a wide wavelength range. This dye is insoluble in water as evidenced by the lack of an absorption corresponding to the dye molecule in Figure 1a. However, in the presence of the amphiphilic polymers 1-5 $\left(10^{-4} \mathrm{M}\right.$ at $\left.\mathrm{pH} 10\right)$, the dye can be solubilized in water as exemplified in Figure 1. This result indicates that the micronevironment provided by the polymer assembly is hydrophobic in nature and is capable of sequestering lipophilic guest molecules, such as RD ( $\sim 5.5$ moles / per mole of $\mathbf{5})$. The absorption maximum of $\mathrm{RD}$ in the presence of the polymer $\mathbf{5}$ is $502 \mathrm{~nm}$. The corresponding $\mathrm{ET}_{30}$ value of 56.9 indicates that the micellar micronevironment is comparable to that of glycerol, which is typical of surfactant-based assemblies. ${ }^{8}$ The $\lambda_{\max }$ obtained for polymers 1 - 4 were similar. These micellar assemblies also exhibit the ability to sequester other lipophilic guest molecules, such as orange-OT.

Similarly, in order to investigate the encapsulation properties of the inverted micelle-type assembly from these polymers in apolar solvents, we used rose bengal (RB) as the spectroscopic probe. $\mathrm{RB}$ is insoluble in toluene. However, in the presence of the amphiphilic polymer, $\left(10^{-4} \mathrm{M}\right)$ significant amount of RB is present in the toluene solution, as evident from the absorption spectra (Figure 1b).

As mentioned above, we have been interested in investigating the behavior of these amphiphilic assemblies in a heterogeneous mixture of solvents. To probe the micelle-type assembly, we added dichloromethane to an aqueous solution containing the polymer 5 with RD sequestered in it. The resulting mixture was shaken for about a minute and allowed to phase separate. It was noted that all the sequestered $\mathrm{RD}$ has been released in to the dichloromethane layer, as noted from Figures $2 \mathrm{a}$ and $2 \mathrm{~b}$. The change in color of the solution from Figures $2 \mathrm{a}$ to $2 \mathrm{~b}$ is due to the solvatochromic nature of RD. ${ }^{8}$ Similarly, water was added to a toluene solution containing the hydrophilic dye molecule, RB, sequestered in the inverse micelle-type assembly of 5. Upon shaking the mixture, the dye molecule was fully transferred from the toluene solution to the aqueous layer as shown in Figures $2 \mathrm{c}$ and $2 \mathrm{~d}$. The significant change in the intensity of color between the solutions in Figures $2 \mathrm{c}$ and $2 \mathrm{~d}$ could be due to the inherently different extinction coefficients of RB in these two environments.

Three limiting mechanistic possibilities can be proposed for the dye release from a micelletype assembly: (i) the distribution coefficient of the polymer dictates the partition in the aqueous phase and the organic phase as micelle-type and inverted micelle-type assemblies respectively. The dye molecule gets released during the process of this thermodynamically driven distribution of the host. (ii) The polymer assembly disintegrates by disassembling at the interface of the two solvents and therefore looses the container property. (iii) The amphiphilic assemblies are stable in the solvent they are assembled in. Thus in water for example, the lipophilic dye molecule is partitioned between the micellar interior and the bulk water. The partition coefficient favors the micellar interior. However, the small concentration of the dye in water is sufficient to transfer the lipophilic dye from the micellar interior to water and then to the apolar solvent.

To distinguish these possibilities, we carried out a simple set of experiments. The micellar assembly of $\mathbf{5}$ was formed in water and then mixed with toluene. The resultant heterogeneous mixture was shaken for a while and then allowed to phase separate. The two layers were analyzed for the presence of the polymer. The polymers were retained in the water layer and no measurable amount of polymer could be seen in the toluene layer (Figure 3a). When a similar 
experiment was carried out with the inverted micelle-type assembly in toluene, the polymer was fully retained in the toluene layer, as shown in Figure 3a. Similar results were obtained in the presence of the dye molecules as well. It is also to be noted that the observed behavior is independent of the lipophilicity of the polymer 1-5. In the mechanisms $(i)$ and (ii), the polymer has the opportunity to find a thermodynamic distribution between the two solvents and therefore the partition coefficient should be independent of the starting point. Thus, the results above rule out the first two mechanistic possibilities and are consistent with the third. It is also interesting to note that these assemblies exhibit very little change in size after subjecting to the heterogeneous mixture (from DLS study). ${ }^{7}$

Considering this behavior of the polymers in an immiscible mixture of solvents, the possibility of using these assemblies in separations based on electrostatics-based host-guest interactions exists. This is conceivable especially because, we had observed that the positively charged dye molecule, rhodamine-6G (R6G), does not get released from the negatively charged inverse micelle (Figures 2e and 2f). Accordingly, a toluene solution of polymer $\mathbf{5}$ was added to an aqueous solution containing a mixture of R6G and RB. After shaking the two solutions for a short time, both layers became colored (Figure $2 \mathrm{~g}$ and $2 \mathrm{~h}$ ). Analyzing the toluene layer and aqueous layer revealed that the polymer 5 was able to selectively extract R6G out of the aqueous layer, while leaving behind RB in water (Figure 3b). To a first approximation from the absorption spectra, the separation seems to be quantitative. Similar separation was also observed when both dye molecules were sequestered into the inverse micelle in toluene and then washed with water (Figure $2 \mathrm{i}$ and $2 \mathrm{j}$ ).

In summary, we have shown that: (i) the micelle and inverse micelle-type assemblies exhibit nanocontainer properties. (ii) These containers are stable in the solvent in which they are assembled in, even in the presence of another immiscible, but favorable solvent. (iii) The mechanism of releasing the sequestered guest molecules is due to the inherent partitioning of the guests between the micellar interior and the bulk solvent. This property indicates that these polymers have potential as controlled drug release vehicles. (iv) The selective binding properties of these assemblies could be used in separations. Further explorations of these polymers for applications in drug delivery and separations are underway in our laboratories.

\section{Supplementary Material}

Refer to Web version on PubMed Central for supplementary material.

\section{Acknowledgements}

Partial support for this work from NIH-NIGMS (GM-65255), Army Research Office and the NSF-funded MRSEC at UMass are gratefully acknowledged.

\section{References}

1. (a) Lehn JM. Angew Chem Int Ed 1988;27:89. (b) Cram DJ. Angew Chem Int Ed 1988;27:1009.

2. Tanford, C. The Hydrophobic Effect. Wiley Interscience; New York: 1973. (b) Blokzij1 W, Engberts JBFN. Angew Chem Int Ed 1993;32:1545.

3. For a few examples, see: (a) Jeong B, Bae YH, Lee DS, Kim SW. Nature 1998;388:860. [PubMed: 9278046] (b) Liu L, Li C, Li X, Yuan Z, An Y, He B. J Appl Polym Sci 2001;80:1976. (c) Kellermann M, Bauer W, Hirsch A, Schade B, Ludwig K, Bottcher C. Angew Chem Int Ed 2004;43:2959.

4. For a few examples, see: (a) Menger FM, Tsuno T, Hammond GS. J Am Chem Soc 1990;112:1263. (b) Sunder A, Kramer M, Hanselmann R, Malhaupt R, Frey H. Angew Chem Int Ed 1999;38:3552. (c) Jung HM, Price KE, McQuade DT. J Am Chem Soc 2003;125:5351. [PubMed: 12720448] (d) Meier MAR, Gohy JF, Fustin CA, Schubert US. J Am Chem Soc 2004;126:11517. [PubMed: 15366897] 
5. Basu S, Vutukuri DR, Shyamroy S, Britto SS, Thayumanavan S. J Am Chem Soc 2004;126:9890. [PubMed: 15303841]

6. Hawker CJ, Bosman AW, Harth E. Chem Rev 2001;101:3661. [PubMed: 11740918]

7. See Supporting Information for details.

8. Reichardt C. Chem Rev 1994;94:2319. 
a)

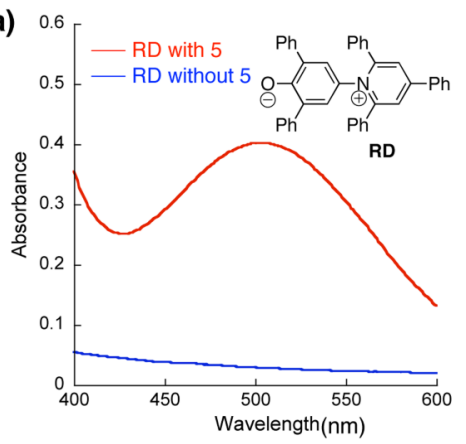

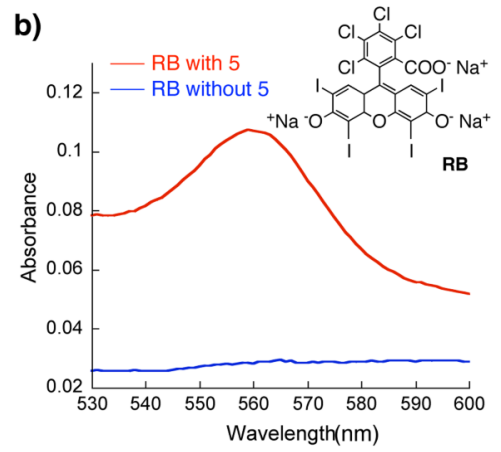

Figure 1.

a) Reichardt's dye in water in the presence and absence of 5. b) Rose Bengal (RB) in toluene in the presence and absence of $\mathbf{5}$. 

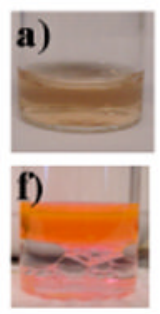
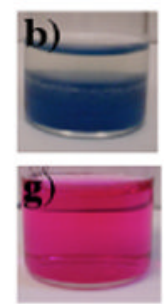

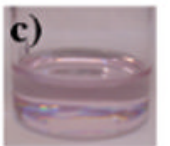

h)

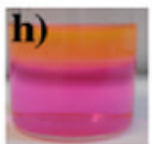

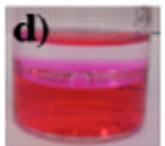

i)

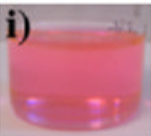

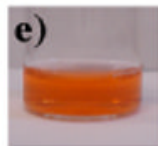

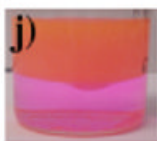

Figure 2.

Extraction studies of dye molecules. a) RD in aqueous solution of 5. b) The dye transferred into DCM (lower layer). c) RB in toluene solution of 5. d) RB released into water (lower layer). e) R6G in toluene solution of 5. f) R6G not released into water (lower layer). g) and i) Mixture of RB and R6G in water and toluene solution of $\mathbf{5}$, respectively. h) R6G separated into toluene solution of $\mathbf{5}$ (upper layer). j) RB separated from the mixture of i) into water (lower layer). 

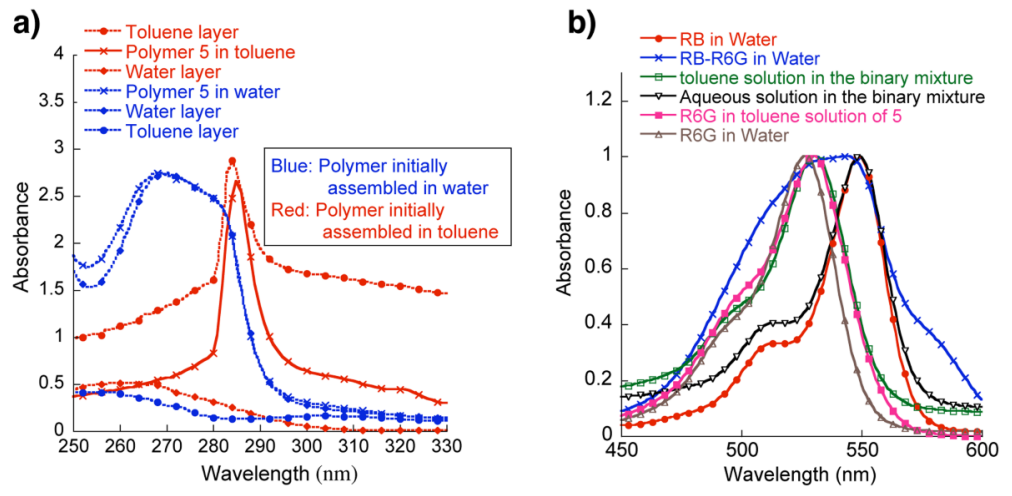

Figure 3.

a) Spectra of polymer $\mathbf{5}$ in aqueous and toluene layers of a binary mixture after it is assembled in one of the solvents. Blue: assembled in water; Red: assembled in toluene. b) Normalized spectra of the selective extraction studies of polymer $\mathbf{5}$ in toluene from a mixture of RB and R6G in water. 


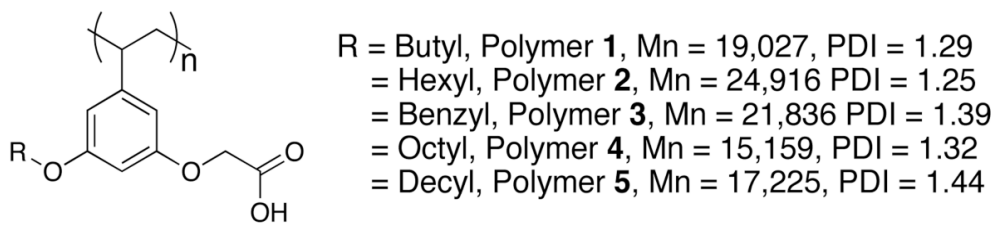

Chart 1.

Structures of the amphiphilic homopolymers in this study. 